\title{
Ecologia humana e antropometria nutricional de adultos Xavánte, Mato Grosso, Brasil
}

\author{
Human ecology and nutritional anthropometry \\ of adult Xavánte Indians in Mato Grosso, Brazil
}

Sílvia A. Gugelmin 1

Ricardo Ventura Santos 2,3

\footnotetext{
1 Departamento de Nutrição Social, Instituto de Nutrição, Universidade do Estado do Rio de Janeiro. Rua São Francisco Xavier 524 , bloco D, sala 12001 Rio de Janeiro, $R J$ 20550-013, Brasil. sigugel@terra.com.br 2 Departamento de Endemia Samuel Pessoa, Escola Nacional de Saúde Pública, Fundação Oswaldo Cruz. Rua Leopoldo Bulhões 1480, Rio de Janeiro, $R J$ 21041-210, Brasil. santos@ensp.fiocruz.br

3 Departamento de Antropologia, Museu Nacional, Universidade Federal do Rio de Janeiro. Quinta da Boa Vista s/no. Rio de Janeiro, $R J$ 20940-040, Brasil.
}

\begin{abstract}
This study compares anthropometric and ecological profiles of two Xavánte indigenous communities in Mato Grosso, Central Brazil. The research describes time allocation patterns and involves an anthropometric survey (including body mass, stature, and BMI) in adults over 20 years of age. Data from Etéñitépa (also known as Pimentel Barbosa) were collected in 1994. Field work at São José was conducted in 1998 and 1999. Compared with the São José group, Xavánte in Etéñitépa do more subsistence activities like farming, fishing, hunting, and gathering. The São José Xavánte do more paid work and generally engage in less physical activity. Average stature in the two communities is similar, but there are major differences in mean body mass and BMI. The São José group has average BMI values well over those of the Etéñitépa group in practically all age brackets. Obesity prevalence rates were high in both men (24.6\%) and women (41.3\%) in São José, while in Etéñitépa the rates were only 2.5\% and 4.8\%, respectively. The authors conclude that the different nutritional profiles in the two communities result from specific patterns of social, political, and economic interactions with Brazilian society.
\end{abstract}

Key words Anthropometry; Obesity; Xavánte; South American Indians

Resumo Este estudo visa analisar comparativamente o perfil ecológico-humano e o antropométrico de duas comunidades indígenas Xavánte - Etéñitépa (ou Pimentel Barbosa) e São Joséde Mato Grosso, Brasil. Os dados foram coletados respectivamente em 1994 e 1998/1999. A pesquisa envolveu caracterização dos padrões de alocação de tempo e de antropometria de adultos acima de 20 anos de idade. Os Xavánte de Etéñitépa despendem mais tempo em atividades ligadas à horticultura, pesca, caça e coleta; os de São José exercem mais funções remuneradas e extra-aldeia, em que há, em geral, menor atividade física. Os valores médios de estatura das duas comunidades são próximos, mas há diferenças marcantes quanto à massa corporal e ao IMC (Índice de Massa Corporal). O grupo de São José exibiu valores de IMC superiores aos de Etéñitépa em quase todas as faixas etárias. Em São José há prevalência de obesidade em homens (24,6\%) $e$ mulheres (41,3\%); em Etéñitépa, os valores foram respectivamente de 2,5\% e 4,8\%. Sugere-se que o perfil nutricional diferenciado resulta, em muito, de diferentes trajetórias de interação social, política e econômica com a sociedade nacional envolvente.

Palavras-chave Antropometria; Obesidade; Xavánte; Índios Sul-Americanos 


\section{Introdução}

O presente estudo tem como objetivo analisar comparativamente aspectos associados ao perfil ecológico-humano e antropométrico - sobretudo quanto a parâmetros relacionados a sobrepeso e à obesidade - de duas comunidades indígenas Xavánte de Mato Grosso. As comunidades apresentam importantes similaridades em relação ao habitat ocupado, à cultura e à organização social. Não obstante, exibem percursos históricos diferenciados de interação com a sociedade nacional brasileira, o que tem repercutido nos fatores ecológico-humanos reconhecidamente associados à situação nutricional, tais como: atividades de subsistência, disponibilidade de recursos naturais, padrões alimentares e mobilidade.

O perfil de saúde-doença dos povos indígenas no Brasil é pouco conhecido, o que decorre da exigüidade de investigações e da precariedade dos sistemas de registro de informações de morbimortalidade (Coimbra Jr. \& Santos, 2000). Da mesma forma, são particularmente escassos os dados relativos à situação nutricional. Essa dimensão merece maior atenção, em virtude de o estado nutricional ser influenciado, em larga medida, por dinâmicas de transformação econômicas, sociais e culturais, assim como, em especial, por alterações em estratégias de subsistência, consumo de alimentos e padrão de atividade física. Tais mudanças são relativamente comuns no cotidiano de grupos indígenas em processo de interação com a sociedade nacional envolvente (Gross et al., 1979; Santos, 1993; Santos \& Coimbra Jr., 1998; Santos et al., 1997; Wirsing, 1985).

Há estudos que apontam para a ocorrência de déficits de crescimento físico em crianças indígenas, o que, em associação a outros parâmetros de morbimortalidade, tem sido usualmente interpretado como manifestação da ocorrência de elevadas prevalências de desnutrição energética-protéica em algumas dessas populações (Coimbra Jr. \& Santos, 1991; Gugelmin, 1995; Leite, 1998; Martins \& Menezes, 1994; Santos, 1993). Concomitantemente, têm surgido na literatura estudos que apontam tanto para significativo incremento nas prevalências de sobrepeso e obesidade em adultos indígenas quanto para a emergência de enfermidades crônicas não-transmissíveis associadas. Assim, elevado aumento de peso em adultos TupiMondé de Rondônia foi evidenciado por Santos \& Coimbra Jr. (1996). Tavares et al. (1999) relatam a ocorrência de casos de intolerância à glicose e diabetes mellitus entre os Parakatejé, do Pará, além de terem observado que $68 \%$ da população adulta apresentava sobrepeso ou obesidade. Estudos realizados por Vieira-Filho (1977, 1981, 1996) e Vieira Filho et al. (1983) reportam o surgimento de diabetes mellitus tipo II ou não-insulino dependente entre os Xavánte, Karipúna e Palikúr. As mudanças no perfil antropométrico-nutricional dos adultos e a emergência de morbimortalidade relacionada a doenças crônicas não transmissíveis têm sido atribuídas a alterações no estilo de vida desses povos.

A literatura concernente à saúde das populações indígenas situadas nas Américas - em particular, aquelas localizadas no Canadá e nos Estados Unidos - aponta para a emergência da obesidade e de complicações associadas - hipertensão arterial, diabetes mellitus tipo II, litíase biliar, entre outras - como sério problema de saúde pública ao longo das últimas décadas, superando inclusive, em certos contextos, as doenças infecciosas e parasitárias em termos de morbimortalidade (Narayan, 1996; Szathmary, 1994; Weiss et al., 1984; Young, 1993, 1994). Ainda que esta não seja a situação dos povos indígenas no Brasil, maior atenção deve ser dada às doenças crônicas não transmissíveis, uma vez que podem vir a tornar-se importante problema de saúde a médio e a longo prazo, sobrepondo-se a um cenário epidemiológico ainda dominado pelas doenças infecto-parasitárias (Coimbra Jr. \& Santos, 1994; Salzano \& Callegari-Jacques, 1988).

\section{Material e Métodos}

\section{População}

Os Xavánte totalizam cerca de 8.000 indivíduos e estão distribuídos em seis Terras Indígenas (TI) localizadas no leste do Estado de Mato Grosso (Lopes-da-Silva, 1992; Ricardo, 1996; Souza \& Santos, 1999). Seus territórios são dominados pela vegetação denominada cerrado. Os Xavánte são afiliados aos tronco lingüístico Macro-Jê. Antes do contato permanente, distribuíam-se em grupos econômica e politicamente autônomos, que desenvolviam economia bimodal e períodos de dispersão seguidos de agregação em grandes aldeias ao longo do ciclo anual. Os Xavánte construíam suas aldeias - e muitos subgrupos ainda constroem na proximidade de matas de galeria, onde cultivavam milho, feijão e abóbora, entre outros cultígenos. Durante vários meses do ano envolviam-se em expedições de caça e coleta, que cobriam extensas áreas e demandavam longas caminhadas. (Flowers, 1983a, 1983b; Giaccaria \& Heide, 1972; Maybury-Lewis, 1967). 
Com o estabelecimento do contato permanente nas décadas de 1940 e 50 - resultado de intensos esforços governamentais no sentido de abrir as regiões do Brasil Central a atividades de colonização - os Xavánte experimentaram sucessivos surtos epidêmicos, redução de territórios e progressiva sedentarização. Ao mesmo tempo, incorporaram novas técnicas de cultivo de alimentos e aumentaram a dependência quanto à agricultura. No final da década de 1970, a Fundação Nacional do Índio (FUNAI) implementou projetos em diversas áreas Xavánte visando à instauração da rizicultura mecanizada ("Projeto Xavánte”). O arroz veio a tornar-se a base da alimentação em diversas comunidades (Flowers, 1983a,b; Graham, 1995; Gugelmin, 1995; Lopes da Silva, 1992; Santos et al., 1997).

Os dados analisados neste estudo foram coletados em duas Terras Indígenas: Pimentel Barbosa (aproximadamente 13o 20' S, 510 40' W, na aldeia Etéñitépa ou Pimentel Barbosa, com 313 habitantes e 23 domicílios em 1994) e Sangradouro-Volta Grande (por volta de 150 50' S, 530 60' W, na aldeia São José, com 590 habitantes e 62 domicílios em 1998). Os Xavánte de Etéñitépa têm sido apontados por antropólogos como um dos mais "tradicionais" (Graham, 1995:37). Ao contrário de outros grupos Xavánte, estes conseguiram manter-se nas mesmas áreas que ocupavam à época do contato, embora seu território tenha sido bastante reduzido e tenham experimentado sucessivos surtos epidêmicos que resultaram em abrupto decréscimo populacional (Flowers, 1983a, 1994; Maybury-Lewis, 1967). Mesmo nos dias atuais, as atividades de caça e coleta de alimentos silvestres permanecem relativamente intensas entre os Xavánte de Etéñitépa (Gugelmin, 1995; Santos et al., 1997), o que deriva, em parte, de condições ecológicas próprias à reserva. Em 1994, uma população de 841 indivíduos vivia na TI Pimentel Barbosa, em território de 328.966 hectares, o que equivale a um quociente de 391,2 hectares/pessoa, o mais elevado dentre todas as áreas Xavánte (Flowers et al., 1998).

Já a trajetória dos Xavánte de SangradouroVolta Grande foi distinta. Na década de 50, com suas terras ocupadas crescentemente por fazendeiros, bem como enfraquecidos pelas doenças, perseguidos e ameaçados, buscaram refúgio junto à Missão Salesiana - pertencente a Igreja Católica. Perderam suas terras por ocasião do contato e nunca as recuperaram plenamente. A TI Sangradouro-Volta Grande ocupa uma extensão de 100.280 hectares, está contígua à Missão Salesiana e, em 1999, contava com população total de 870 indivíduos, ou seja, 115,3 hectares/pessoa, o que é cifra bastante inferior àquela verificada para Pimentel Barbosa. Em decorrência da pressão populacional, a disponibilidade de recursos naturais em Sangradouro-Volta Grande tem sido reportada como menos pronunciada que em outras áreas Xavánte (Leite, 1998). Ainda que a relação hectare/pessoa não possa ser tomada como medida absoluta e confiável quanto à disponibilidade de recursos ambientais, guarda estreita relação com depredação ambiental, intensidade de uso dos solos e pressão sobre caça, pesca e coleta, entre outros, que são dimensões estreitamente vinculadas à pressão demográfica.

\section{Metodologia}

A pesquisa envolveu dois componentes básicos: estudo ecológico-humano baseado na caracterização dos padrões de alocação de tempo e inquérito antropométrico-nutricional de adultos. A população-alvo constituiu-se de indivíduos de ambos os sexos com idade acima de 20 anos. Os dados relativos à Etéñitépa foram coletados em maio/junho e novembro/dezembro de 1994, tendo sido parcialmente analisados anteriormente (Gugelmin, 1995; Santos et al., 1997). A pesquisa de campo na aldeia São José foi conduzida em setembro/outubro de 1998 e janeiro/fevereiro de 1999.

O componente ecológico-humano visou primordialmente a identificar diferenças entre as duas comunidades no que se refere à utilização do tempo - em particular, quanto às atividades de subsistência. A metodologia proposta por Johnson (1975) e Gross (1984) foi seguida. Dois dias da semana foram sorteados aleatoriamente para as visitas, distribuídas em dois horários por dia e abrangendo três domicílios para cada horário. Todos os domicílios de ambas as comunidades fizeram parte do sorteio. As visitas eram realizadas entre às 7:00 e 18:00 horas, ocasião em que foram registradas as atividades que cada membro do domicílio estava realizando, além de sua idade, sexo e horário específico. Se havia alguém ausente, perguntava-se aos parentes presentes onde estava e que atividade estaria realizando. Posteriormente, as informações eram conferidas.

Os dados antropométricos - massa corporal e estatura - foram coletados nas casas dos indivíduos, utilizando-se, como instrumentos, uma balança de plataforma marca Seca - com capacidade máxima de $150 \mathrm{~kg}$ e precisão de $0,5 \mathrm{~kg}$ - e um antropômetro metálico marca GPM, com precisão de $1 \mathrm{~mm}$. As pessoas foram pesadas e medidas com roupas leves e descalças. 
O intuito da investigação antropométrica foi incluir o maior número possível de indivíduos. Em Etéñitépa foram medidos 90 adultos - na análise, oito gestantes foram excluídas -, o equivalente a $83,3 \%$ da população total. Em São José foram medidos 134 indivíduos - seis gestantes foram excluídas -, ou seja, 69,4\% da população adulta total. Em ambas as comunidades, a maior parte das perdas foi de indivíduos que estavam ausentes de seus domicílios no momento da coleta de dados, ou dos que não se mostraram interessados em participar do estudo.

Para o cômputo das idades dos Xavánte de São José, foram empregados os dados demográficos de Souza (1999) e Souza \& Santos (1999), bem como os registros da FUNAI e da Missão Salesiana. Quanto à Etéñitépa, utilizaram-se as informações demográficas resultantes das investigações conduzidas por Flowers (1994).

$\mathrm{O}$ projeto de pesquisa que gerou esta investigação foi aprovado pelo Comitê de Ética da Escola Nacional de Saúde Pública, da Fundação Oswaldo Cruz, e submetido posteriormente à Comissão Nacional de Ética em Pesquisa (CONEP), tendo sido aprovado. Foi apreciado também pela Fundação Nacional do Índio (FUNAI) e pelas lideranças indígenas Xavánte.

\section{Análise}

Os dados de alocação de tempo foram codificados e organizados em seis categorias excludentes. Tal classificação baseou-se naquela usada anteriormente por Flowers (1983a) e Gugelmin (1995) em investigações com os Xavánte. As atividades foram assim agrupadas: (1) subsistência: inclui trabalho na roça, caça, pesca e coleta de alimentos e produtos silvestres; (2) doméstica: inclui atenção a crianças, preparo de alimentos, manutenção e limpeza do domicílio, manufatura de peças em geral - artesanato, brinquedos, utilitários etc.; (3) remunerada: inclui o exercício de atividades como professor, merendeira, secretário da escola, motorista, trabalhador em fazendas, funcionário da FUNAI, entre outras; (4) estudo: inclui atividades relacionadas à ida a escola, a trabalhos escolares; (5) extra-aldeia: diz respeito à ausência dos indivíduos da aldeia com vistas a tratamento médico, viagens, estudo, idas a centros urbanos e a outras aldeias; (6) pessoal/outras: inclui as demais atividades, em particular aquelas de caráter pessoal, tais como: alimentação, higiene, repouso, visitas, recreação, entre outras.

Para os valores da massa corporal $(\mathrm{kg})$, de estatura $(\mathrm{cm})$ e do índice de massa corporal
(IMC) (massa corporal em kg dividida pela estatura em metro ao quadrado), foram calculados médias e desvios padrões. Na distribuição por categorias de IMC foram empregados os seguintes pontos de corte, recomendados pela WHO (1998): baixo peso: < 18,5kg.m-2; adequado: 18,5 a 24,9kg.m-2; sobrepeso: 25,0 a 29,9 kg.m-2; obesidade: $\geq 30,0 \mathrm{~kg} \cdot \mathrm{m}^{-2}$.

As análises estatísticas foram realizadas através dos programas Epi Info, versão 6.01 (WHO, 1994) e SPSS for Windows (SPSS Incorporation, 1993). Empregaram-se o teste do quiquadrado na comparação de proporções e o teste t-Student ou a análise de variância para comparar os valores médios das variáveis contínuas. Aceitou-se um valor de p igual ou menor que 0,05 como nível de significância.

\section{Resultados}

Na investigação acerca da alocação de tempo, foram feitas 502 observações em Etéñitépa e 668 em São José, distribuídas aproximadamente de maneira eqüitativa entre homens e mulheres (Tabela 1). Detectaram-se diferenças estatisticamente significativas nos conjuntos de distribuições das freqüências de atividades entre as duas comunidades $(\mathrm{p}<0,05)$. Em ambas, a categoria mais comum foi pessoal/outras, que correspondeu, em Etéñitépa, a mais da metade dos casos (55\%) e, em São José, por volta de $41 \%$ do total. Há notável similaridade no que diz respeito ao tempo alocado para as atividades domésticas, próximas de $20 \%$ em ambas. Para as demais categorias, observa-se uma série de diferenças ao serem contrastadas as duas comunidades. Os Xavánte de Etéñitépa gastam maior tempo em atividades de subsistência, quais sejam, em trabalho na roça, caça, pesca e coleta de alimentos silvestres. Em contrapartida, o grupo de São José encontra-se proporcionalmente mais envolvido em funções remuneradas, atividades relacionadas à escola e extra-aldeia. Para estas três últimas categorias, há nítido predomínio por parte do sexo masculino, o que faz sentido à luz do padrão de divisão sexual do trabalho entre os Xavánte. Em seu conjunto, os dados sugerem que o grupo de Etéñitépa encontra-se mais engajado em ocupações que envolvem maiores níveis de atividade física se comparado ao grupo de São José.

A Tabela 2 exibe maior detalhamento quanto às freqüências das atividades relacionadas a trabalho, o que confirma as observações do parágrafo anterior. Os resultados dizem respeito aos sexos combinados, dado que os tamanhos de amostra são relativamente pequenos. Há ní- 
Distribuição absoluta e relativa das atividades desenvolvidas pelos adultos Xavánte de Etéñitépa e São José (1994, 1998/1999), a partir da análise de alocação de tempo.

\begin{tabular}{lrrrrrr}
\hline Atividades & Homens & $\begin{array}{c}\text { Etéñitépa } \\
\text { Mulheres }\end{array}$ & \multicolumn{1}{c}{ Total } & Homens & $\begin{array}{r}\text { São José } \\
\text { Mulheres }\end{array}$ & Total \\
\hline Subsistência & $41(17,0)$ & $33(12,6)$ & $74(14,7)$ & $43(12,3)$ & $18(5,7)$ & $61(9,1)$ \\
Doméstica & $20(8,3)$ & $85(32,6)$ & $105(20,9)$ & $27(7,7)$ & $115(36,3)$ & $142(21,3)$ \\
Remunerada & $05(2,1)$ & - & $05(1,0)$ & $32(9,1)$ & $07(2,2)$ & $39(5,8)$ \\
Estudo & - & - & - & $19(5,4)$ & $03(1,0)$ & $22(3,3)$ \\
Extra-aldeia & $33(13,7)$ & $07(2,7)$ & $40(8,0)$ & $90(25,6)$ & $42(13,2)$ & $132(19,8)$ \\
Pessoal/Outras & $142(58,9)$ & $136(52,1)$ & $278(55,4)$ & $140(39,9)$ & $132(41,6)$ & $272(40,7)$ \\
Total & 241 & 261 & 502 & 351 & 317 & 668 \\
& $(100,0 \%)$ & $(100,0 \%)$ & $(100,0 \%)$ & $(100,0 \%)$ & $(100,0 \%)$ & $(100,0 \%)$ \\
\hline
\end{tabular}

$\chi^{2}=82,45 ; 5$ g.l.; $p=0,00$ (comparação entre Etéñitépa e São José, sexos combinados).

tidas diferenças entre as duas comunidades. Em Etéñitépa, agricultura e caça/pesca/coleta sobrepujam as atividades relacionadas a trabalho remunerado. Observa-se padrão inverso em São José.

A Tabela 3 compara as médias de estatura (EST), massa corporal (MC) e índice de massa corporal (IMC) dos adultos de Etéñitépa com os dados oriundos de São José. Se os valores médios de EST das duas comunidades encontram-se bastante próximos, há marcantes diferenças quanto à MC e ao IMC. Os homens de São José apresentam média de MC superior em $5,1 \mathrm{~kg}$ em relação aos seus pares de Etéñitépa; para as mulheres, a diferença atinge $12,6 \mathrm{~kg}$. Em decorrência, as médias do IMC são consideravelmente superiores em São José, da ordem de duas unidades para os homens e de cinco unidades para as mulheres.

Os homens e mulheres de São José apresentam médias de IMC superiores àquelas dos Xavánte de Etéñitépa em praticamente todas as faixas etárias (Figura 1). A partir dos 40 anos, nota-se tendência de diminuição dos valores de IMC - em especial, entre as mulheres.

A distribuição relativa das categorias do IMC está exposta na Tabela 4. Em São José, chamam a atenção as elevadas prevalências de sobrepeso e obesidade que, juntas, somam $78 \%$ para os sexos combinados. A obesidade é mais pronunciada entre as mulheres $(41,3 \%)$ de São José. A maioria dos Xavánte de Etéñitépa recaem nas categorias "normal" e "sobrepeso". O baixo peso é praticamente inexistente em ambas as comunidades.

\section{Tabela 2}

Distribuição absoluta e relativa das atividades de subsistência e trabalho remunerado desenvolvidos pelos adultos Xavánte de Etéñitépa e São José, sexos combinados (1994, 1998/1999).

\begin{tabular}{lrr}
\hline Atividades & Etéñitépa & São José \\
\hline Agricultura & $44(55,7)$ & $29(29,0)$ \\
Caça, pesca e coleta & $30(38,0)$ & $32(32,0)$ \\
Trabalho remunerado & $05(6,3)$ & $39(39,0)$ \\
Total & $79(100,0 \%)$ & $100(100,0 \%)$ \\
\hline
\end{tabular}

$\chi^{2}=27,33 ; 2$ g.l. $; p=0,00$.

\section{Discussão}

Os resultados desta investigação apontam para importantes diferenças em aspectos ecológicohumanos e no perfil antropométrico entre os dois grupos Xavánte investigados. Os adultos de Etéñitépa, que é a comunidade na qual a subsistência baseada na agricultura, caça, pesca e coleta é praticada com maior intensidade e cuja área da reserva é maior, o que potencialmente se traduz em maior disponibilidade de recursos naturais, apresentam valores médios de massa corporal (MC) e do índice de massa corporal (IMC) significativamente menores que seus pares de São José. Já nesta comunidade, a subsistência encontra-se mais distanciada do padrão "tradicional" Xavánte, o que repercute na intensidade da atividade física. Os Xavánte de São José estão proporcionalmente mais envolvidos em atividades relacionadas a trabalho remunerado, as quais demandam geralmente in- 
Médias de estatura (EST), massa corporal (MC) e índice da massa corporal (IMC) da população adulta Xavánte ( $\geq 20$ anos) de Etéñitépa e São José, Mato Grosso, Brasil.

\begin{tabular}{|c|c|c|c|c|c|c|c|c|}
\hline \multirow[t]{2}{*}{ Comunidades } & \multicolumn{4}{|c|}{ Homens } & \multicolumn{4}{|c|}{ Mulheres } \\
\hline & $\mathrm{n}$ & $\begin{array}{l}\text { EST } \\
(\mathrm{cm})\end{array}$ & $\begin{array}{l}\mathrm{MC} \\
(\mathrm{Kg})\end{array}$ & $\begin{array}{c}\text { IMC } \\
(\mathrm{kg} \cdot \mathrm{m}-2)\end{array}$ & $n$ & $\begin{array}{l}\text { EST } \\
(\mathrm{cm})\end{array}$ & $\begin{array}{c}\mathrm{MC} \\
(\mathrm{Kg})\end{array}$ & $\begin{array}{c}\text { IMC } \\
\left(\mathrm{kg} \cdot \mathrm{m}^{-2}\right)\end{array}$ \\
\hline Etéñitépa & 40 & 167,3 & 70,4 & 25,2 & 42 & 154,6 & 59,0 & 24,6 \\
\hline São José & 65 & 166,8 & 75,5 & 27,1 & 63 & 155,3 & 71,6 & 29,7 \\
\hline Teste t-Student & & $p=0,65$ & $p=0,01$ & $p=0,01$ & & $p=0,39$ & $p=0,00$ & $P=0,00$ \\
\hline
\end{tabular}

Figura 1

Distribuição das médias do índice de massa corporal (IMC), segundo grupos etários e sexo, para os Xavánte de Etéñitépa e São José, Mato Grosso, Brasil.

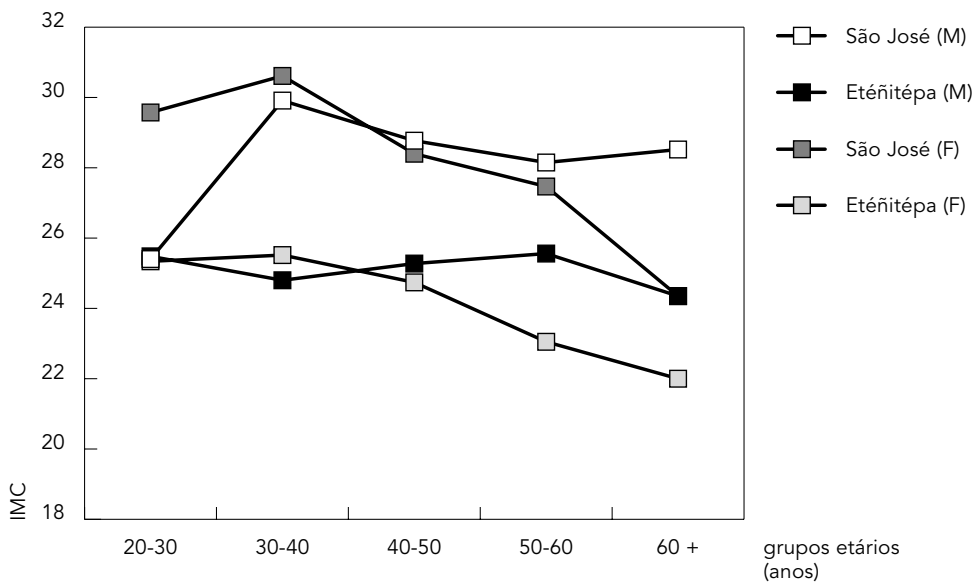

tensidade de atividade física menos pronunciada.

Ainda que seja possível conjeturar acerca dos determinantes, individuais e coletivos, concernentes à expressão da obesidade entre os Xavánte, não é possível apontá-los com precisão tendo, por fundamento, os dados disponíveis e, muito menos, hierarquizá-los e/ou quantificá-los. São múltiplos e complexos os fatores envolvidos, incluindo padrões de consumo alimentar, intensidade de atividade física, entre outros. Não obstante, é útil relatar alguns dos fatores possivelmente relacionados à ocorrência de obesidade, notadamente no caso de São José. O recebimento de aposentadorias, salários e outras fontes de renda, bem como maior percentagem de tempo despendido fora da al- deia, potencialmente se traduzem em menor engajamento nas atividades de subsistência, aspecto evidenciado pela investigação a respeito da alocação de tempo (Tabela 1). A localização geográfica de São José - distante apenas $50 \mathrm{~km}$ da cidade de Primavera do Leste -, a facilidade de transporte e a existência de energia elétrica são aspectos que também devem estar influenciando hábitos alimentares e o estilo de vida do grupo. Afora isso, os adultos de São José estão proporcionalmente mais envolvidos em atividades eminentemente sedentárias, como, por exemplo, lecionar, trabalhar na secretaria e na inspetoria da escola, conduzir caminhão, trator ou carro de menor porte. Essas tarefas demandam menor gasto energético se comparadas àquelas associadas à subsistência baseada na agricultura, caça, pesca e coleta. Durante o trabalho de campo em São José, ainda que não tenha sido possível realizar inquérito detalhado acerca do consumo alimentar, observou-se que a dieta era dominada sobretudo por alimentos amiláceos, parcela deles industrializados, tais como, entre outros, arroz polido, macarrão, farinha de mandioca recebidos do programa governamental Comunidade Solidária ou adquiridos nos mercados da região -, pão, biscoitos e refrigerantes (Leite, 1998; Vieira-Filho, 1981, 1996). No início da década de 1980, Vieira-Filho (1981) chamou a atenção para a redução da diversificação alimentar, além da predominância de dieta rica em amido e pobre em proteínas de origem animal, em Sangradouro-Volta Grande e em algumas outras áreas Xavánte, situação que aparentemente se tornou mais aguda com o tempo.

A situação descrita acima para São José apresenta importantes diferenças se contrastada com o que se observa em Etéñitépa. Ainda que os Xavánte de Etéñitépa tenham experimentado profundas mudanças em seus padrões de subsistência em anos recentes, ainda dependem cotidianamente, em larga medi- 
da, de recursos extraídos da reserva (Flowers, 1983a, 1983b; Graham, 1995; Gugelmin, 1995; Santos et al., 1997). Por ocasião da coleta de dados, não havia luz elétrica na comunidade, a cidade mais próxima localizava-se a $100 \mathrm{~km}$ de distância e, no entorno da aldeia, eram encontradas áreas de cerrado e mata ciliar relativamente preservadas, que permitiam a realização de atividades agrícolas, caça, pesca e coleta de alimentos silvestres (ver também Gugelmin, 1995). Em investigação a respeito do consumo alimentar, realizada na comunidade de Etéñitépa em 1994, Santos et al. (1997) verificaram que, de um total de 840 episódios de consumo de alimentos, apenas $11 \%$ envolviam alimentos industrializados (ver também Gugelmin, 1995).

As últimas décadas têm sido de mudanças em diversas esferas para os Xavánte, inclusive no que diz respeito à ecologia humana e à nutrição. De modo não surpreendente, o perfil antropométrico dos adultos de Etéñitépa e de São José distancia-se daquele relatado nas investigações efetuadas nas comunidades Xavánte em décadas anteriores. Nos anos 60, Neel et al. (1964) e Niswander et al. (1967) coletaram dados antropométricos entre os Xavánte de São Domingos e Simões Lopes, quando obtiveram valores de MC de 67,2 e $69,8 \mathrm{~kg}$ para homens e 54,0 e 57,9kg para mulheres, respectivamente. Quanto à estatura, foram observadas médias de 168,1 e 170,2cm para homens e de 154,7 e $156,3 \mathrm{~cm}$ para mulheres. Ainda que seja necessário cautela - em razão, sobretudo, das diferenças metodológicas - ao se comparar dados coletados anteriormente, fruto de inquéritos conduzidos nos anos 90 (Tabela 3), com aqueles reportados neste trabalho, um padrão relativamente consistente emerge de análises comparativas. Se os valores médios de estatura dos Xavánte referidos por Neel et al. (1964) e Niswander et al. (1967) pouco diferem daqueles de Etéñitépa e São José nos anos 90 (a propósito, os valores mais antigos são ligeiramente mais elevados), há diferenças marcantes e consistentes no que diz respeito à MC. Os homens de Etéñitépa apresentam médias de massa corporal superiores em 0,6-3,2kg em relação aos dados da década de 60; para os de São José, a diferença alcança $5,7-8,3 \mathrm{~kg}$. As diferenças são ainda maiores para as mulheres: $1,1-5,0 \mathrm{~kg}$ para as de Etéñitépa e 13,7-17,6kg para as de São José. Em outras palavras, o conjunto de informações sugere que as amostras Xavánte medidas nos anos 90 exibem médias de MC mais elevadas do que as de seus pares investigados três décadas antes. A magnitude das diferenças é mais pronunciada para os Xavánte de São José.

\begin{tabular}{|c|c|c|c|c|}
\hline \multicolumn{5}{|c|}{$\begin{array}{l}\text { Distribuição absoluta e relativa de categorias do índice de massa } \\
\text { corporal (IMC) na população adulta Xavánte ( } \geq 20 \text { anos) de Etéñitépa } \\
\text { e São José, Mato Grosso, Brasil. }\end{array}$} \\
\hline \multirow[t]{2}{*}{ Categorias } & \multicolumn{2}{|c|}{ Etéñitépa } & \multicolumn{2}{|c|}{ São José } \\
\hline & $\mathrm{n}$ & $\%$ & $\mathrm{n}$ & $\%$ \\
\hline \multicolumn{5}{|l|}{ Homens } \\
\hline Baixo peso 1 & - & - & 01 & 1,5 \\
\hline Adequado2 & 20 & 50,0 & 21 & 32,3 \\
\hline Sobrepeso 3 & 19 & 47,5 & 27 & 41,6 \\
\hline Obesidade 4 & 01 & 2,5 & 16 & 24,6 \\
\hline \multicolumn{5}{|l|}{ Mulheres } \\
\hline Baixo peso 1 & - & - & - & - \\
\hline Adequado2 & 22 & 52,4 & 06 & 9,5 \\
\hline Sobrepeso 3 & 18 & 42,8 & 31 & 49,2 \\
\hline Obesidade 4 & 02 & 4,8 & 26 & 41,3 \\
\hline
\end{tabular}

$1 \mathrm{IMC}<18,5 \mathrm{~kg} \cdot \mathrm{m}-2 ; 218,5 \leq \mathrm{IMC} \leq-24,9 \mathrm{~kg} \cdot \mathrm{m}-2$;

$325,0 \leq \mathrm{IMC} \leq 29,9 \mathrm{~kg} \cdot \mathrm{m}^{-2} ; 4 \mathrm{IMC} \geq 30,0 \mathrm{~kg} \cdot \mathrm{m}-2$

Não se pode dizer, contudo, que ocorreu tendência secular positiva em estatura.

Há outras evidências a sugerir que, de fato, está em marcha uma tendência de aumento de massa corporal entre os adultos Xavánte. Em 1976/77, o mesmo grupo de Etéñitepá investigado neste trabalho foi estudado por Flowers (1983a, 1983b). Esta autora observou que os homens adultos apresentavam médias MC de 66,0kg e as mulheres, de 57,7kg (Flowers, 1983b: 374). Portanto, em intervalo de aproximadamente vinte anos, os valores médios aumentaram em $4,4 \mathrm{~kg}$ nos homens e $1,3 \mathrm{~kg}$ nas mulheres de Etéñitépa.

A freqüência de adultos Xavánte residentes em São José com IMC superior a 30 - considerados obesos - é extremamente elevada, da ordem de 33\% (Tabela 4). Em Etéñitépa, ainda que parcela expressiva dos adultos (45\%) apresente valores de IMC entre 25 e 30 , o que os classificaria como portando sobrepeso, somente $4 \%$ recaem na faixa de IMC tida como obesidade. Dados resultantes de inquéritos de representatividade nacional realizados no Brasil durante os últimos anos - como aqueles oriundos da Pesquisa Nacional sobre Saúde e Nutrição (PNSN), conduzida em 1989 - apontam para a prevalência de obesidade na população brasileira por volta de 9,6\% em adultos entre 25 a 64 anos (Monteiro et al., 1995). Por conseguinte, mesmo limitando a amostra Xavánte para a faixa etária entre 25 a 64 anos, a prevalência de obesidade entre os Xavánte de São José é bem mais elevada (39,6\%) que aque- 
la de Etéñitépa $(4,2 \%)$, e sobrepuja, em muito, os valores para a população brasileira, na qual a tendência tem sido de aumento de prevalências nas últimas décadas (Monteiro et al., 1995, 2000).

Elevadíssimas freqüências de obesidade e patologias associadas são importantes problemas de saúde pública em grupos indígenas da América do Norte - em particular, no Canadá e nos Estados Unidos (Narayan, 1996; Szathmary, 1994; Weiss et al., 1984; Young, 1993, 1994). Em povos indígenas situados no Brasil, apesar de o problema ainda não atingir as proporções verificadas em seus congêneres norte-americanos, há crescente número de estudos que apontam para a emergência da obesidade, diabetes mellitus tipo II, doenças cardiovasculares, entre outras, em certos grupos que têm em comum o fato de estarem experimentando profundas alterações em seus sistemas de subsistência, dieta e padrões de atividade física (Santos \& Coimbra Jr., 1996; Tavares et al., 1999; Vieira-Filho, 1981, 1996). A propósito, há relatos de que a diabetes já se faz presente em SangradouroVolta Grande, onde está localizada a aldeia São José. Vieira-Filho (1996) menciona que há pelo menos cinco casos de diabetes não-insulino dependente em Sangradouro-Volta Grande. Nessa mesma comunicação, o autor sumariza da seguinte maneira suas observações clínicoepidemiológicas, resultantes de duas décadas de interação com os Xavánte: "Quando iniciei as minhas visitas anuais aos índios Xavántes de
Sangradouro e São Marcos há 20 anos, observei que eram delgados e com atividade física intensa, não havendo nenhum caso com sintomatologia de diabetes melito... Nos últimos anos, têm ocorrido casos de diabetes com sintomatologia exuberante entre os Xavántes que se tornaram obesos" (Vieira-Filho, 1996:61).

Em conclusão, os dados apresentados neste trabalho evidenciam diferenças no perfil ecológico-humano, analisado através da alocação de tempo, e no perfil antropométrico das duas populações estudadas. Em Etéñitépa, que é a comunidade na qual a subsistência ainda se baseia, em larga medida, na agricultura, caça, pesca e coleta, os adultos apresentam valores menores de massa corporal e do índice de massa corporal. São José, por sua vez, encontra-se mais distanciada desse padrão tanto em relação à alimentação como no que diz respeito aos padrões de atividade física, o que, em parte, deve estar associado à restrição territorial. Os Xavánte de São José estão proporcionalmente mais envolvidos em atividades relacionadas a trabalho remunerado, que encerram menor intensidade de atividade física. As prevalências de obesidade em São José superam inclusive as médias para a população brasileira. As diferenças encontradas entre as duas comunidades relacionam-se, em larga medida, a distintas trajetórias de interação com a sociedade nacional envolvente, manifestas por amplas implicações potenciais no perfil saúde/ doença.

\section{Agradecimentos}

Aos Xavánte de Etéñitépa e São José que nos acolheram e permitiram a realização de nosso trabalho; ao Programa de Apoio à Pesquisa Estratégica em Saúde/Fundação Oswaldo Cruz e Fundação Nacional de Saúde/Centro Nacional de Epidemiologia/VIGISUS pelo apoio financeiro. 


\section{Referências}

COIMBRA Jr., C. E. A. \& SANTOS, R. V., 1991. Avaliação do estado nutricional num contexto de mudança sócio-econômica: O grupo indígena Suruí do estado de Rondônia, Brasil. Cadernos de Saúde Pública, 7:538-562.

COIMBRA Jr., C. E. A. \& SANTOS, R. V., 1994. Epidemiologic Profile of Amazonian Amerindians from Brazil, with Special Emphasis on the Xavánte from Mato Grosso and on Groups from Rondônia. A Report to the World Bank. Rio de Janeiro: World Bank. (mimeo.)

COIMBRA Jr., C. E. A. \& SANTOS, R. V., 2000. Saúde, minorias e desigualdade: Algumas teias de interrelações, com ênfase nos povos indígenas. Ciência \& Saúde Coletiva, 5:125-132.

FLOWERS, N. M., 1983a. Forager-farmers: The Xavánte Indians of Central Brazil. Dissertação de Doutorado, New York: City University of New York.

FLOWERS, N. M., 1983b. Seasonal factors in subsistence, nutrition, and child growth in a Central Brazilian Indian Community. In: Adaptative Responses of Native Amazonians (R. B. Hames \& W. H. Vickers, eds.), pp. 357-390, New York: Academic Press.

FLOWERS, N. M., 1994. Crise e recuperação demográfica: Os Xavánte de Pimentel Barbosa, Mato Grosso. In: Saúde e Povos Indígenas (C. E. A. Coimbra Jr. \& R. V. Santos, org.), pp. 213-242, Rio de Janeiro: Editora Fiocruz.

FLOWERS, N. M.; GUGELMIN, S. A. \& SANTOS, R. V., 1998. Settlement pattern as economic and political strategy: The Xavánte of Central Brazil. South American Indian Studies, 5:18-28.

GIACCARIA, B. \& HEIDE, A., 1972. Xavánte (Auwe Uptabi: Povo Autêntico). São Paulo: Editorial Dom Bosco.

GRAHAM, L. R., 1995. Performing Dreams: Discourses of Immortality among the Xavánte of Central Brazil. Austin: University of Texas Press.

GROSS, D. R., 1984. Time allocation: A tool for the study of cultural behavior. Annual Review of Anthropology, 13:519-558.

GROSS, D. R.; EITEN, G.; FLOWERS, N. M.; RITTER, M. \& WERNER, D., 1979. Ecology and acculturation among native people of Central Brazil. Science, 206:1043-1050.

GUGELMIN, S. A., 1995. Nutrição e Alocação de Tempo dos Xavánte de Pimentel Barbosa, Mato Grosso: Um Estudo em Ecologia Humana e Mudanças. Dissertação de Mestrado, Rio de Janeiro: Escola Nacional de Saúde Pública, Fundação Oswaldo Cruz.

JOHNSON, A., 1975. Time allocation in a Machiguenga community. Ethnology, 14:301-310.

LEITE, M. S., 1998. Avaliação do Estado Nutricional da População Xavánte de Sangradouro-Volta Grande, Mato Grosso. Dissertação de Mestrado, Rio de Janeiro: Escola Nacional de Saúde Pública, Fundação Oswaldo Cruz.

LOPES-DA-SILVA, A., 1992. Dois séculos e meio de história Xavánte. In: História dos Índios no Brasil (M. C. da Cunha, org.), pp. 357-378, São Paulo: Companhia das Letras.

MARTINS, S. J. \& MENEZES, R. C., 1994. Evolução do estado nutricional de menores de 5 anos em aldeias indígenas da tribo Parakanã, na Amazônia Oriental Brasileira (1989-1991). Revista de Saúde Pública, 28:1-8.

MAYBURY-LEWIS, D., 1967. Akwe-Shavante Society. Oxford: Clarendon Press.

MONTEIRO, C. A.; MONDINI, L.; SOUZA, A. L. M. \& POPKIN, B. M., 1995. Da desnutrição para a obesidade: A transição nutricional no Brasil. In: $V e$ lhos e Novos Males da Saúde no Brasil (C. A. Monteiro, org.), pp. 247-255, São Paulo: Editora Hucitec.

MONTEIRO, C. A.; BENÍCIO, M. H., CONDE, W. L. \& POPKIN, B. M., 2000. Shifting obesity trends in Brazil. European Journal of Clinical Nutrition, 54:342-346.

NARAYAN, K. M. V., 1996. Diabetes mellitus in Native Americans: The problem and its implications. In: Changing Numbers, Changing Needs (G. D. Sandefur, R. R. Rindfuss \& B. Cohen, ed.), pp. 262-288, Washington: National Academy Press.

NEEL, J. V.; SALZANO, F. M.; JUNQUEIRA, P. C.; KEITER, F. \& MAYBURY-LEWIS, D., 1964. Studies on the Xavánte Indians of the Brazilian Mato Grosso. American Journal of Human Genetics, 16:52-139.

NISWANDER, J. D.; KEITER, F. \& NEEL, J. V., 1967. Further studies on the Xavánte Indians. II. Some anthropometric, dematoglyphic, and nonquantitative morphological traits of the Xavántes of Simões Lopes. American Journal of Human Genetics, 19:490-501.

RICARDO, C. A., 1996. A sociodiversidade nativa contemporânea no Brasil. In: Povos Indígenas no Brasil 1991/1995 (C. A. Ricardo, org.), pp. 1-12. São Paulo: Instituto Socioambiental.

SALZANO, F. M. \& CALLEGARI-JACQUES, S. M., 1988. South American Indians: A Case Study in Human Evolution. Oxford: Clarendon Press.

SANTOS, R. V., 1993. Crescimento físico e estado nutricional de populações indígenas brasileiras. Cadernos de Saúde Pública, 9(Sup. 1):46-57.

SANTOS, R. V. \& COIMBRA Jr., C. E. A., 1996. Socioeconomic differentiation and body morphology in the Suruí of Southwestern Amazonia. Current Anthropology, 37:851-856.

SANTOS, R. V. \& COIMBRA Jr., C. E. A., 1998. On the (un)natural history of the Tupí-Mondé Indians: Bioanthropology and change in the Brazilian Amazon. In: Building a New Biocultural Synthesis: Political-economic Perspectives on Human Biology (A.H. Goodman \& T. Leatherman, ed.), pp. 269-294, Ann Arbor: University of Michigan Press.

SANTOS, R. V.; FLOWERS, N. M.; COIMBRA Jr., C. E. A. \& GUGELMIN, S. A., 1997. Tapirs, tractors, and tapes: The changing economy and ecology of the Xavánte Indians of Central Brazil. Human Ecology, 25:545-566.

SOUZA, L. G., 1999. Perfil Demográfico dos Xavánte de Sangradouro - Volta Grande, 1993-1997. Dissertação de Mestrado, Rio de Janeiro: Escola Nacional de Saúde Pública, Fundação Oswaldo Cruz.

SOUZA, L. G. \& SANTOS, R. V., 1999. Mortalidade, Fecundidade e Padrão de Assentamentos dos Xavánte de Sangradouro - Volta Grande, Mato Grosso (1993- 
1997). Documento de Trabalho no 2. Porto Velho: Universidade de Rondônia, Centro de Estudos em Saúde do Índio de Rondônia/Rio de Janeiro: Escola Nacional de Saúde Pública, Fundação Oswaldo Cruz.

SPSS INCORPORATION, 1995. SPSS for Windows. Statistical Package for the Social Sciences. Release 7.0. Chicago: SPSS Inc.

SZATHMÁRY, E. J. E., 1994. Non-insulin dependent diabetes mellitus among aboriginal North Americans. Annual Review of Anthropology, 23:457-482.

TAVARES, E. F; VIEIRA FILHO, J. P. B.; ANDRIOLO, A \& FRANCO, L. J., 1999. Anormalidades de tolerância à glicose e fatores de risco cardiovascular em uma tribo indígena aculturada da região Amazônica brasileira. Arquivos Brasileiros de Endocrinologia e Metabologia, 43(Sup. 1):s235.

VIEIRA FILHO, J. P. B., 1977. O diabetes mellitus e as glicemias de jejum dos índios Caripuna e Palikur. Revista da Associação Médica Brasileira, 23:175178.

VIEIRA FILHO, J. P. B., 1981. Problemas da aculturação alimentar dos Xavántes e Bororo. Revista de Antropologia, 24:37-40.

VIEIRA FILHO, J. P. B., 1996. Emergência do diabetes melito tipo II entre os Xavántes. Revista da Associação Médica Brasileira, 42:61.
VIEIRA FILHO, J. P. B.; RUSSO, E. M. K. \& NOVO, N. F., 1983. A hemoglobina glicosilada (HbAl) dos índios Xavántes. Arquivos Brasileiros de Endocrinologia e Metabologia, 27:153-156.

WEISS, K. M.; FERRELL, R. E. \& HANIS, C. L., 1984. A New World syndrome of metabolic diseases with a genetic and evolutionary basis. Yearbook of Physical Anthropology, 27:153-178.

WHO (World Health Organization), 1994. Epi Info. Version 6.01. A Word Processing, Database, and Statistics System for Epidemiology on Microcomputers. Geneva: WHO.

WHO (World Health Organization), 1998. Obesity. Preventing and Managing the Global Epidemic. Report of WHO Consultation on Obesity. Geneva: WHO.

WIRSING, R. L., 1985. The health of traditional societies and the effects of acculturation. Current Anthropology, 26:303-322.

YOUNG, T. K., 1993. Diabetes mellitus among Native Americans in Canada and the United States: An epidemiological review. American Journal of $\mathrm{Hu}$ man Biology, 5:399-413.

YOUNG, T. K., 1994. The Health of Native Americans. Toward a Biocultural Epidemiology. New York: Oxford University Press. 